\title{
Tumor suppressor microRNA-18a regulates tumor proliferation and invasion by targeting TBPL1 in colorectal cancer cells
}

\author{
GUANGHUI LIU*, YUANHUA LIU*, ZHEN YANG, JUNXIA WANG, DONGYAN LI and XIEFU ZHANG \\ Department of Gastrointestinal Surgery, The First Affiliated Hospital of Zhengzhou University, \\ Zhengzhou, Henan 450052, P.R. China
}

Received November 19, 2014; Accepted August 14, 2015

DOI: $10.3892 / \mathrm{mmr} .2015 .4335$

\begin{abstract}
Recent advances in the understanding of microRNA have rendered microRNAs (miRNAs) a compelling novel class of biomarker in cancer biology. However, the specific function of miRNA-18a (miR-18a) in colorectal cancer (CRC) remains unclear. In the present study, the role of miR-18a in the carcinogenesis of CRC was investigated. miR-18a expression was assessed in CRC specimens and cell lines using reverse transcription-quantitative polymerase chain reaction (RT-qPCR). The targets of miR-18a were predicted using bioinformatics tools. Luciferase reporter assays were used to confirm the functional association between miR-18a and its target genes. The effect of miR-18a on cell proliferation, invasion and migration was confirmed in vitro by a methylthiazol tetrazolium assay, cell invasion assay, and wound healing assay. Gene and protein expression was examined using RT-qPCR and western blotting, respectively. It was demonstrated that the expression of miR-18a in CRC tissues and cell lines was markedly lower than in normal control tissues and cells, respectively. In addition, miR-18a inhibited cell proliferation, invasion and migration in CRC cells. Moreover, TATA box-binding protein-like protein 1 (TBPL1) was identified as a potential target gene of miR-18a in the bioinformatics analysis and luciferase reporter assays, and miR-18a directly inhibited TBPL1 expression by targeting its 3'-untranslated region. Furthermore, TBPL1 was downregulated and inversely correlated with miR-18a expression in tissues. These findings demonstrate that miR-18a exhibits a protective role in CRC via inhibiting proliferation, invasion and migration of CRC cells by directly targeting the TBPL1 gene.
\end{abstract}

Correspondence to: Dr Xiefu Zhang, Department of Gastrointestinal Surgery, The First Affiliated Hospital of Zhengzhou University, 1 Jianshe Road, Zhengzhou, Henan 450052, P.R. China E-mail: xiefu_zhang@126.com

*Contributed equally

Key words: colorectal cancer, microRNA-18a, invasion, metastasis, TATA box-binding protein-like protein 1

\section{Introduction}

Colorectal cancer (CRC) is one of the most common types of malignant cancer and is the third leading cause of cancer-associated mortality worldwide (1). As is common with all cancers, the overwhelming cause of fatality in patients with CRC is metastasis, a complex series of multiple sequential steps during which primary cancer cells acquire an invasive phenotype enabling them to translocate from the primary tumor to a distant organ and form a secondary tumor (2). Tumor invasion and metastasis are parts of a complicated process in which the tumor grows, then detaches from the primary site and metastasizes to a distant organ. Aberrations in protein-coding genes have been widely accepted to be critical in the pathology of CRC, including oncogenes and tumor suppressor genes (3). The underlying molecular mechanism of metastasis is yet to be elucidated. Thus, there is a growing need to understand the molecular pathogenesis underlying CRC. Over the last decade, microRNAs (miRNAs) have emerged as key players in carcinogenesis. Aberrant expression of miRNAs has been demonstrated to be critical in the initiation and progression of several types of human cancer through post-transcriptional regulation of gene expression (4-6).

miRNAs are emerging as regulators of metastasis by acting on multiple signaling pathways (7). miRNAs are noncoding RNAs of 18-24 nucleotides that inhibit translation or induce mRNA decay through binding to the 3'-untranslated regions (3'-UTRs) of their target RNAs $(8,9)$, influencing processes such as tumor cell proliferation, differentiation, apoptosis and invasion $(4,7,10)$. Recently, a series of studies has revealed that miRNAs regulate various genes that are pivotal in the process of tumor progression $(11,12)$. It has specifically been shown that miRNAs are important in the development of CRC. In CRC miRNAs exhibit oncogenic or tumor suppressive roles by directly regulating oncogenes or tumor-suppressor genes $(13,14)$. Several studies have indicated the role of miRNAs in regulating tumor invasion and metastasis in CRC. For example, miR-133a represses tumor growth and metastasis in CRC by targeting LIM and SH3 protein 1 (LASP1) and inhibiting the mitogen-activated protein kinase pathway (15). Additionally, miR-132 inhibits CRC invasion and metastasis via directly targeting ZEB2 (16). miR-103/107 promotes metastasis of CRC by targeting the metastasis suppressors Death-associated protein kinase (DAPK) and Kruppel-like factor 4 (KLF4) (17). 
However, miR-18a has instead been reported to suppress cell proliferation in T24 bladder cancer cells (18). Recently, the diagnostic value of circulating miR-18a in the plasma was reported for pancreatic cancer (19). Therefore, miR-18a may exhibit different roles in various types of cancer through different targeted genes. However, the role of miR-18a in CRC progression and metastasis remains largely unclear.

The present study aimed to examine the role of miR-18a in the progression of CRC, and, to clarify the molecular mechanism by which miR-18a promotes malignant tumor progression. Efforts to elucidate the association between miR-18a and TBPL1 are also included in the present study. In addition, the present study aimed to provide a novel miRNA and target gene for cancer therapy.

\section{Materials and methods}

Cell lines and clinical specimens. Human SW620 and HCT116 CRC cell lines were obtained from the Cell Bank of the Type Culture Collection (Shanghai, China). All cell lines were cultured in Dulbecco's modified Eagle's medium (DMEM) supplemented with $10 \%$ fetal bovine serum (Gibco Life Technologies, Carlsbad, CA, USA) in a humidified $37^{\circ} \mathrm{C}$ incubator supplemented with $5 \% \mathrm{CO}_{2}$. The medium was changed every 2-3 days, and the cells were trypsinized (Mingzhu Chemical Co., Shanghai, China) when $80-90 \%$ confluence was reached.

Twenty-five CRC tissue samples and adjacent normal colon tissue samples were collected directly after surgical resection at the First Affiliated Hospital of Zhengzhou University (Zhengzhou, China). The NCM460 normal colonic epithelial cell lines were purchased from the American Type Culture Collection (Manassas, VA, USA). All patients had a histological diagnosis of CRC. Following resection, all specimens were immediately frozen in liquid nitrogen and stored at $-80^{\circ} \mathrm{C}$ until RNA extraction. Approval for this study was obtained from the Ethics Review Committee of the Institutional Review Board of the First Affiliated Hospital of Zhengzhou University and informed consent was obtained from each patient.

RNA isolation and RT-qPCR analysis. Total RNA from the tissues and cells was extracted using TRIzol reagent (Invitrogen Life Technologies, Carlsbad, CA, USA) according to the manufacturer's instructions. RNA quality and concentration were determined using the Nanodrop 2000 system (Thermo Fisher Scientific, Wilmington, NC, USA). To analyze gene expression levels, $5 \mu \mathrm{g}$ RNA was reverse transcribed to cDNA using a PrimeScript RT Reagent kit (Takara Biotechnology Co., Ltd., Dalian, China). Mature miR-18a and U6 levels were quantified with TaqMan miRNA assays (Applied Biosystems, Foster City, CA, USA). TBPL1 and $\beta$-actin mRNA levels were determined by RT-qPCR using the SYBR Green Master mix on the HT 7500 System (Applied Biosystems). The PCR primers for TBPL1 were 5'-CCTCTTCCCACGGATGTGAT-3' (sense) and 5'-GAGTCCAATGTGCAGCAGT-3' (reverse). The qPCR cycle was as follows: $98^{\circ} \mathrm{C}$ for $2 \mathrm{~min}, 40$ cycles of $95^{\circ} \mathrm{C}$ for $15 \mathrm{~s}$ and $60^{\circ} \mathrm{C}$ for $30 \mathrm{~s}$. The final melting curve analysis $\left(60-95^{\circ} \mathrm{C}\right)$ was included. The relative expression levels of each gene were calculated and normalized using the $2^{-\Delta \Delta \mathrm{Ct}}$ method relative to U6 or $\beta$-actin. All of the reactions were run in triplicate.
Western blot analysis. Cells were lysed with radioimmunoprecipitation assay lysis buffer containing protease inhibitors (Roche, Indianapolis, IN, USA). Protein concentrations were determined using a Pierce Bicinchoninic Acid Protein Assay kit (Thermo Fisher Scientific, Logan, UT, USA). Equal quantities of proteins were loaded and separated on $10 \%$ SDS-PAGE and then transferred to a polyvinylidene difluoride membrane (Millipore, Billerica, MA, USA). Non-specific protein interactions were blocked by incubation with $3 \%$ fat-free milk in Tris-buffered saline buffer (containing $150 \mathrm{mM} \mathrm{NaCl}$ and $50 \mathrm{mM}$ Tris- $\mathrm{HCl}, \mathrm{pH} 7.6$ ) at $4^{\circ} \mathrm{C}$ for $1 \mathrm{~h}$. The membranes were incubated with the following primary antibodies at $4^{\circ} \mathrm{C}$ overnight: Rabbit anti-human TBPL1 polyclonal antibody (cat. no. ABIN1875056; 1/1,000; Antibodies Online, Atlanta, GA, USA) and mouse anti-human GAPDH monoclonal antibody (sc-365062; 1/2,000; Santa Cruz Biotechnology Inc., Santa Cruz, CA, USA). Unbound antibody was removed by washing in TBS with Tween-20 (Sigma-Aldrich, St. Louis, MO, USA) buffer (TBST) three times (10 min/wash). The membranes were then incubated with horseradish peroxidase-conjugated secondary antibodies (Zhongshan, Beijing, China) at $25^{\circ} \mathrm{C}$ for $1 \mathrm{~h}$, followed by washing with TBST buffer three times (10 min/wash). The blots were developed with the enhanced chemiluminescence reagent (Millipore). GAPDH was used as an internal control.

Oligonucleotide transfection. Cells were transfected with oligonucleotides using Lipofectamine 2000 (Invitrogen Life Technologies) according to the manufacturer's instructions. The miR-18a mimic, miR-18a inhibitor and negative control (NC) oligonucleotides were purchased from Ribobio (Guangzhou, China). The cells were plated in a six-well plate one day prior to transfection. All cell transfections were performed with the miR-18a mimic (miR-18a inhibitor) or NC $(50 \mathrm{nmol} / \mathrm{l})$. Transfections were conducted in triplicate. Cells were observed by inverted microscopy (Nikon TE 300, Nikon Inc., Melville, NY, USA) 24 h after transfection. Oligonucleotide expression was confirmed by RT-qPCR.

Methylthiazol tetrazolium (MTT) assay. An MTT assay was performed to quantify CRC cell viability following transfection with the miR-18a mimic, miR-18a inhibitor or NC. Following incubating the cells for $36 \mathrm{~h}$ at $37^{\circ} \mathrm{C}$ in a humidified $5 \% \mathrm{CO}_{2}$ atmosphere, the cells were stained with $100 \mu \mathrm{l}$ sterile 3-(4,5-dimethythiazol-2-yl)-2,5-diphenyl tetrazolium bromide $\left(0.5 \mathrm{mg} / \mathrm{ml}\right.$; Sigma-Aldrich) for $4 \mathrm{~h}$ at $37^{\circ} \mathrm{C}$. The culture medium was then removed and formazan crystals in the cells were solubilized using dimethyl sulfoxide (Sigma-Aldrich) with shaking of the plate for $30 \mathrm{~min}$. The absorbance was measured at $570 \mathrm{~nm}$ with a spectrophotometer (Bio-Rad Laboratories, Hercules, CA, USA). All experiments were performed in triplicate.

Cell invasion assay. Cell invasion was determined using 24-well Transwell chambers coated with Matrigel (BD Biosciences,

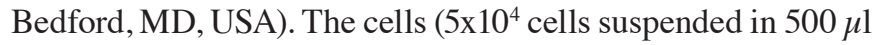
of serum-free medium) were added to the upper chamber of the inserts, which were coated with a Matrigel mix; fetal bovine serum $(500 \mu \mathrm{l})$ was added to the bottom chamber as a chemoattractant. After $24 \mathrm{~h}$, the non-invading cells on the upper surface were removed and the cells that had invaded the bottom side of the membrane were fixed with methanol, 
A

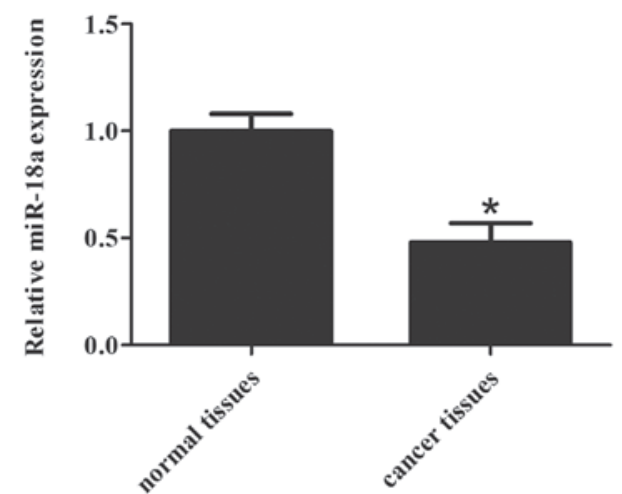

B

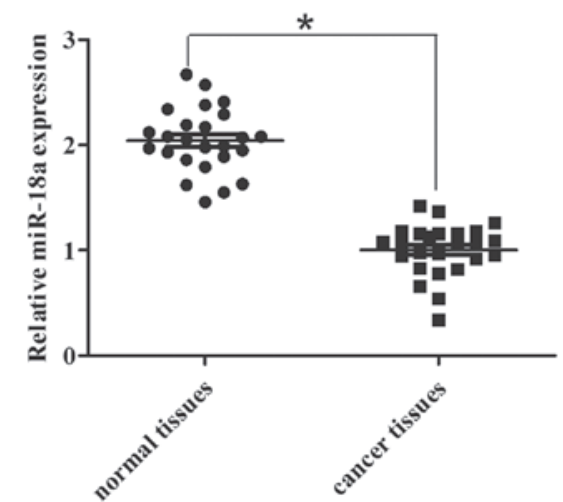

C

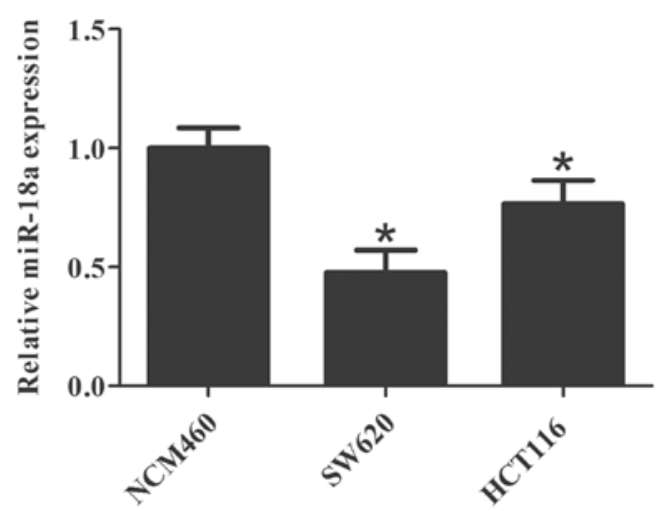

Figure 1. miR-18a is downregulated in CRC. (A) RNAs were isolated from CRC tissues or matched paracarcinoma tissues, and miRNA expression profiles were determined by miRNA microarray. miR-18a expression was normalized to a control consisting of random sequences of a similar size. One representative example from two experiments performed is shown (B) The expression levels of mature miR-18a in CRC tissues and matched normal tissues were determined by RT-qPCR; and (C) The expression levels of miR-18a in two CRC cell lines and the NCM460 normal colonic cell line were confirmed by RT-qPCR. Data are representative of three experiments. Error bars represent mean \pm standard deviation. ${ }^{*} \mathrm{P}<0.05$ vs. normal control. miR, microRNA; CRC, colorectal cancer; RT-qPCR, reverse transcription-quantitative polymerase chain reaction.

stained with $0.1 \%$ crystal violet (Shanghai Chemical Reagent Company, Shanghai, China), air dried and subjected to digital image acquisition Olympus BX51 fluorescence microscope (Olympus Optical Co., Tokyo, Japan). The number of invasive cells was evaluated in five independent fields under a microscope. The mean of triplicate assays for each experimental condition was determined.

Wound healing assay. After transfection with the miR-18a mimic, miR-18a inhibitor or NC $\left(5.0 \times 10^{5}\right.$ cells per well), the cell lines were seeded into 6-well plates (Corning, Corning, NY, USA) for $24 \mathrm{~h}$ and allowed to grow to $90 \%$ confluence. Linear scratch wounds were then created (in triplicate) on the confluent monolayer using a pipette tip and the cells were cultured in the same medium without FBS. Immediately after wounding (time 0 ) and at $48 \mathrm{~h}$, images were captured using a digital camera mounted on a light microscope (Axiophot; Carl Zeiss, Oberkochen, Germany). The width of the wound gap was measured using NIH Image J 1.61 analysis (National Institutes of Health, Bethesda, MA, USA) and normalized to the width at time 0 for four independent experiments.

Vector construction and dual-luciferase reporter assay. The potential miR-18a targets were predicted and analyzed using three publicly available algorithms: PicTar (http://www.pictar. org/cgi-bin/PicTar_vertebrate.cgi), TargetScan (http://www. targetscan.org/) and microRNA.org (http://www.microrna. org/microrna/home.do). The number of false-positive results was decreased by accepting only putative target genes that were predicted by at least two programs.

The $1.77 \mathrm{~kb}$ 3'-untranslated region (UTR) of human TBPL1 was amplified from a human cDNA library using PCR and cloned into a pGL3 Vector (Promega Corporation, Madison, WI, USA) to generate the Wt-pGL3-TBPL1-3'-UTR vector (WT vector). The following primers were used: Forward: $5^{\prime}-\mathrm{CTC}$ TCTAGACTCAAA AGA AAACTGGACCAAC-3'; and reverse: 5'-CTCTCTAGATGCACATTCAATTGAAAA A-3'. Site-directed mutagenesis of the miR-18a target site in the TBPL1 3'-UTR was performed using mutation primers to generate the Mut-pGL3-TBPL1-3'-UTR vector (Mut vector). After cells were plated in a 12-well plate at $\sim 80 \%$ confluence, the experiments were conducted in two parts. Firstly, cells were co-transfected with the WT vector and $30 \mathrm{nM}$ miRNA $\mathrm{NC}$, miR-18a mimic or miR-18a inhibitor. In addition, cells were co-transfected with miR-18a mimic and the pGL3 vector, WT vector or Mut vector. Each sample was also co-transfected with $0.05 \mu \mathrm{g}$ phRL-null plasmid expressing Renilla luciferase (Promega Corporation) as an internal control for transfection efficiency. Cell transfection was performed using Lipofectamine 2000 (Invitrogen Life Technologies) according to the manufacturer's instructions. A luciferase assay was performed $48 \mathrm{~h}$ after transfection using the Dual Luciferase Reporter Assay system (Promega Corporation), and each experiment was repeated in triplicate.

Statistical analysis. Data are expressed as the mean \pm standard deviation from at least three independent experiments. The significance between groups was analyzed using Student's t-test. All statistical analyses were performed using SPSS 13.0 (SPSS, Inc., Chicago, IL, USA). Spearman's correlation test or the $\chi^{2}$ test were also conducted. $\mathrm{P}<0.05$ was considered to indicate a statistically significant difference.

\section{Results}

miR-18a is significantly downregulated in CRC cell lines and CRC tissues. According to the miRNA array data from the patients with CRC, miR-18a was found to be significantly downregulated in CRC tissues compared with their 
A

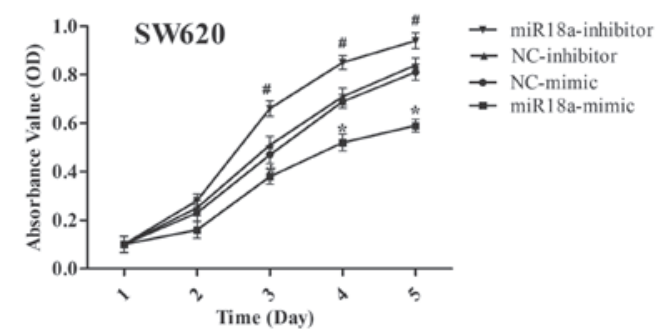

B

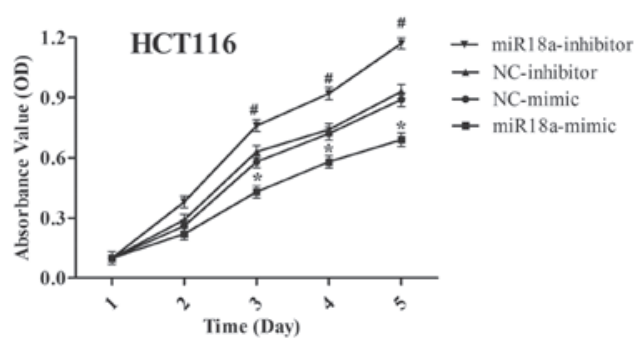

Figure 2. miR-18a inhibits colorectal cancer cell proliferation. Proliferation curves of (A) SW620 and (B) HCT116 cells, respectively. The effect of miR-18a on cell proliferation was evaluated by a methylthiazol tetrazolium assay after miR-18a transfection. miR, microRNA; NC, negative control. ${ }^{*} \mathrm{P}<0.05$, vs. NC-mimic and ${ }^{\#} \mathrm{P}<0.05$, vs. NC-inhibitor.

A

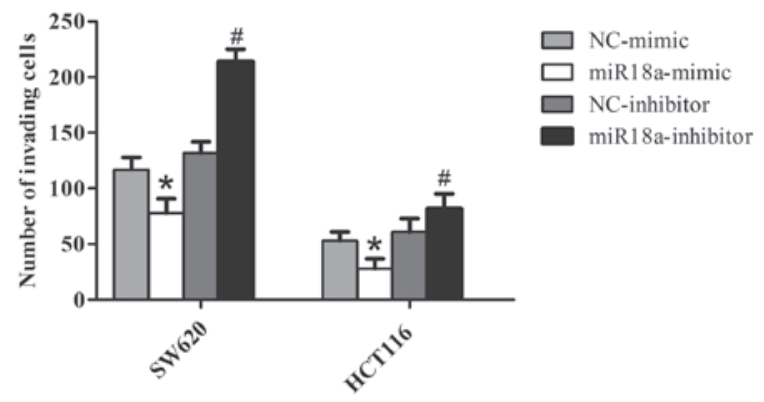

B

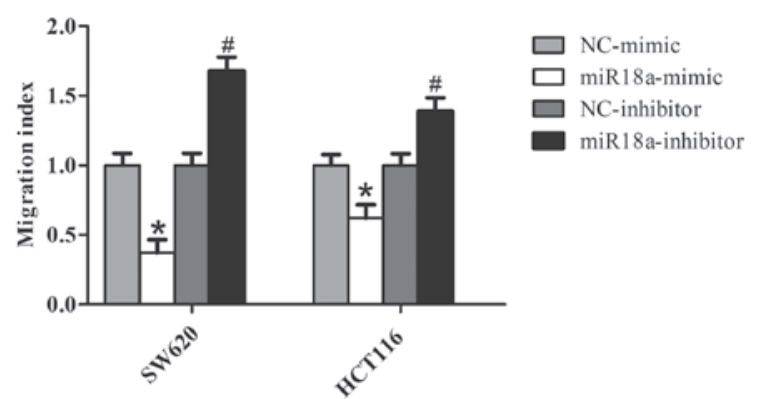

Figure 3. miR-18a inhibits colorectal cancer cell invasion and migration. (A) Data from the Transwell assay for SW620 and HCT116 cells. The cells were counted under a microscope in five randomly selected fields. Bars represent the number of cells invading after transfection with miR-18a. (B) Data from a wound healing assay are shown for SW620 and HCT116 cells. Bars represent the motility index of treatment expressed as a value relative to the number of cells translocating in the miR controls. Each error bar represents the mean \pm standard deviation. The results were reproduced in three independent experiments. ${ }^{*} \mathrm{P}<0.05$ vs. NC-mimic, ${ }^{~} \mathrm{P}<0.05$ vs. NC-inhibitor. miR, microRNA; NC, negative control.

matched normal tissues (Fig. 1A). To confirm this, miR-18a levels were measured in the 25 CRC tissues by RT-qPCR.
The results showed that miR-18a was downregulated in cancer tissues compared with normal controls (Fig. 1B). To further confirm that this phenomenon is consistent and common in CRC cell lines, the expression of miR-18a in two CRC cell lines and a NCM460 normal colonic cell line was detected by RT-qPCR. As shown in Fig. 1C, miR-18a was downregulated in CRC cell lines compared with the NCM460 normal colonic cell line. These data revealed that the expression of miR-18a was universally downregulated in CRC cells and in patients with CRC, indicating that increased miR-18a expression may contribute to the malignant phenotype and development of the tumor.

miR-18a inhibits CRC cell proliferation. To investigate whether miR-18a is involved in the progression of CRC, an MTT assay was conducted to determine the effect of miR-18a on cell proliferation in SW620 and HCT116 cells transiently transfected either with miR-18a mimic or inhibitor. It was revealed that miR-18a inhibited cell proliferation in these two cell lines. As shown in Fig. 2, the increased expression of miR-18a significantly suppressed cell proliferation in SW620 (Fig. 2A) and HCT116 (Fig. 2B) cells $(\mathrm{P}<0.05)$. Correspondingly, after transfection with miR-18a inhibitors, cell proliferation was increased compared with the control group. These findings demonstrate that miR-18a expression significantly reduced the proliferation of SW620 and HCT116 cells.

miR-18a suppresses CRC cell invasion and migration in vitro. In light of the findings described previously, it was hypothesized that miR-18a could inhibit cell invasion by CRC. Two cell lines, SW620 and HCT116, were transfected with the miR-18a mimic to overexpress miR-18a, or transfected with the miR-18a inhibitor to knockdown endogenous miR-18a. As expected, overexpression of miR-18a decreased CRC cell invasion. By contrast, knockdown of miR-18a significantly increased the invasiveness of the CRC cells (Fig. 3A). Similarly, the wound healing assay demonstrated that overexpression of miR-18a markedly reduced the migration of CRC cells, and knockdown of miR-18a in CRC cells increased wound healing (Fig. 3B). These observations suggest that miR-18a significantly promotes in vitro migration and invasion of CRC cells.

TBPL1 is identified as a direct target of miR-18a. It is well-known that miRNAs exert their function through suppressing the expression of their target genes. To better understand the molecular mechanisms of miR-18a, bioinformatics analyses was performed using the miRNA target analysis tools PicTar, TargetScan and microRNA.org in order to predict putative miRNAs binding to the TBPL1 3'-UTR. According to the analysis, all three programs predicted that a binding sequence in the 3'-UTR of TBPL1 was a good match for the miR-18a seed (Fig. 4A). The bioinformatics analysis thus indicated a potential functional link between TBPL1 and miR-18a.

Furthermore, a luciferase reporter assay was performed to further confirm whether miR-18a can directly target the 3'-UTR region of TBPL1 in CRC cells. The TBPL1 wild-type 3'-UTR containing the miR-18a-binding site and a mutated TBPL1 3'-UTR sequence were cloned into modified pGL-3 luciferase reporter vectors, which were co-transfected into 
A

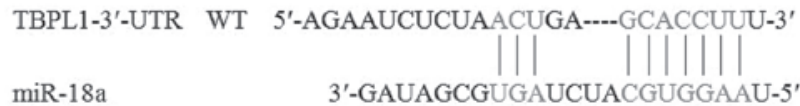

TBPL1 3'-UTR Mut 5'-AGAAUCUCUAUUCUGA----GGACCUAU-3'

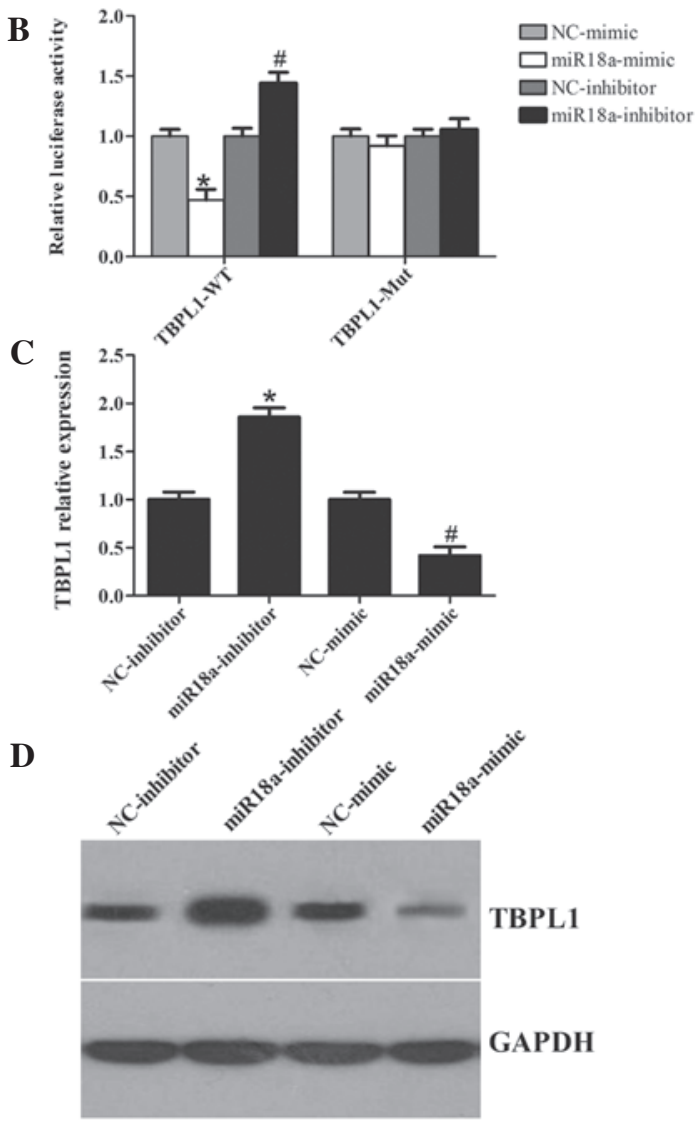

Figure 4. miR-18a directly targets TBPL1 by binding to its 3'-UTR. (A) The predicted miR-18a binding site within the TBPL1 3'-UTR and a mutated version generated by site directed mutagenesis are shown. (B) Luciferase reporter assay illustrating direct binding of miR-18a to the WT, but not MT sequences within the 3'-UTR of TBPL1. (C) mRNA levels of TBPL1 were determined by reverse transcription-quantitative polymerase chain reaction in transfected SW620 cells. (D) Western blot analysis was used to confirm the expression of TBPL1 in SW620 cells after transfection; GAPDH was used as a control. The statistical analysis was based on three independen experiments. Error bars represent the mean \pm standard deviation. ${ }^{*} \mathrm{P}<0.05$ vs. NC-mimic, ${ }^{\prime} \mathrm{P}<0.05$ vs. NC-inhibitor. miR, microRNA; TBPL1, TATA box-binding protein-like protein 1; UTR, untranslated region; WT, wild-type; Mut, mutant.

SW620 cells together with NC, the miR-18a mimic, or the miR-18a inhibitor. As shown in Fig. 4B, miR-18a overexpression significantly reduced the luciferase reporter activity by the TBPL1 3'-UTR in a consistent manner, and inhibition of miR-18a had the opposite effect. However, TBPL1 3'-UTR luciferase reporter activity was unaffected by point mutations in the miR-18a-binding seed region. Collectively, these data suggest that miR-18a may inhibit post-transcriptional TBPL1 expression by targeting its 3'-UTR. As predicted, western blot analysis showed that, at $48 \mathrm{~h}$ after transfection, the enhanced miR-18a in SW620 cells significantly repressed TBPL1 protein expression compared with cells transfected with a scrambled control. By comparison, downregulation of miR-18a by inhibitors in SW620 cells led to a moderate increase in the TBPL1 mRNA level (Fig. 4C). In addition, apparent alterations in
TBPL1 protein expression were also observed (Fig. 4D). Together, these data provide strong evidence that TBPL1 is a specific target of miR-18a in CRC cells.

\section{Discussion}

CRC is a highly malignant tumor that leads to $>600,000$ fatalities worldwide annually (20). Therefore, it is important to investigate and validate novel biomarkers that are involved in CRC development. Deregulation of miRNAs has been observed in various types of human cancer $(21,22)$; however, the molecular mechanisms by which miRNAs modulate carcinogenesis and cancer progression remain unclear. Metastasis is the major reason for a poor prognosis in CRC, and increasing evidence supports the contribution of miRNAs to cancer progression. miRNAs have been found to be involved in the regulation of multiple pathological processes that contribute to tumorigenesis. A number of miRNAs have been identified that regulate tumor metastasis $(23,24)$. Recent studies have increasingly linked microRNAs to CRC. Although aberrant expression of several miRNAs occurs in CRC metastasis, such as miR-7 (25), miR-29a (26), miR-132 (16), miR-103 and miR-107 (17), and miR-143 (27), novel candidates with a definite molecular mechanism that are involved in promoting CRC metastasis require further investigation. The pathological role and regulatory mechanism of miR-18a in the development and progression of colorectal cancer still remain unclear.

In a previous study, overexpression of miR-18a in the plasma of patients with pancreatic cancer as compared with healthy individuals was revealed, suggesting that miR-18a may be involved in pancreatic tumorigenesis (19). Conversely, it was reported that miR-18a* can suppress K-Ras to inhibit tumor growth (28). Recent findings have shown high level expression of miR-18a in breast (29), bladder (18) and pancreatic (19) cancer. In the present study, miR-18a expression was markedly downregulated in CRC cells and clinical tissues samples compared with normal colonic cells and adjacent noncancerous tissues from the same patient, respectively. Until now, no functional evidence of such a role has been documented. The present study aimed to identify the role of miR-18a in CRC by investigating its effects on cell proliferation, migration and invasion. It was demonstrated that overexpression of miR-18a could effectively reduce CRC cell growth, migration and invasion in vitro, whereas knockdown of miR-18a expression promoted CRC cell proliferation, migration and invasion. Although the precise mechanisms are not fully understood, these findings nevertheless suggest that miR-18a may be important in the proliferation of CRC.

Generally, miRNAs function as posttranscriptional repressors that exert their biological function by suppressing their target genes. Each miRNA can potentially downregulate a number of target genes through binding their 3'-UTRs. In the present study, predicted by bioinformatics analysis and validated by luciferase reporter gene assay, TBPL1 was identified as the major regulatory target of miR-18a. Thus, it was deduced that miR-18a may function by interacting with the TBPL1 gene. Consistent with this hypothesis, RT-qPCR and western blot analysis demonstrated that miR-18a could 
decrease the expression of TBPL1 at the mRNA and protein levels. In addition, inhibiting miR-18a expression could increase TBPL1 expression. A luciferase assay also showed that miR-18a could interact with the 3'-UTR of TBPL1. These observations indicate that TBPL1 is a bona fide miR-18a-targeted gene. Further investigations are required to identify and characterize other miR-18a targets in order to fully elucidate the functional role of miR-18a in CRC development, progression and metastasis.

In conclusion, the results showed that miR-18a, acts as an oncogenic miRNA, significantly reducing TBPL1 expression, which subsequently inhibits CRC cell proliferation, migration and invasion by its functional target TBPL1. The results of this study indicate that miR-18a may exhibit a suppressive role in the development and progression of CRC. To the best of our knowledge, this study is the first to reveal a novel gene, TBPL1, as a target for miR-18a in CRC. This may be used as a potential biomarker or target for CRC diagnosis and chemotherapy. Further exploration of the molecular link between miR-18a and TBPL1 may advance our understanding of the intricate molecular regulation of $\mathrm{CRC}$ cells and may provide novel targets for anticancer treatment.

\section{References}

1. Edwards BK, Ward E, Kohler BA, Eheman C, Zauber AG, Anderson RN, Jemal A, Schymura MJ, Lansdorp-Vogelaar I, Seeff LC, et al: Annual report to the nation on the status of cancer, 1975-2006, featuring colorectal cancer trends and impact of interventions (risk factors, screening and treatment) to reduce future rates. Cancer 116: 544-573, 2010 .

2. Chaffer CL and Weinberg RA: A perspective on cancer cell metastasis. Science 331: 1559-1564, 2011

3. Wood LD, Parsons DW, Jones S, Lin J, Sjöblom T, Leary RJ, Shen D, Boca SM, Barber T, Ptak J, et al: The genomic landscapes of human breast and colorectal cancers. Science 318: 1108-1113, 2007.

4. Calin GA and Croce CM: MicroRNA signatures in human cancers. Nat Rev Cancer 6: 857-866, 2006.

5. Croce CM: Oncogenes and cancer. New Engl J Med 358: 502-511, 2008.

6. Deng S, Calin GA, Croce CM, Coukos G and Zhang L: Mechanisms of microRNA deregulation in human cancer. Cell Cycle 7: 2643-2646, 2008.

7. Nicoloso MS, Spizzo R, Shimizu M, Rossi S and Calin GA: MicroRNAs-the micro steering wheel of tumour metastases. Nat Rev Cancer 9: 293-302, 2009.

8. Bartel DP: MicroRNAs: Genomics, biogenesis, mechanism, and function. Cell 116: 281-297, 2004.

9. He L and Hannon GJ: MicroRNAs: Small RNAs with a big role in gene regulation. Nat Rev Genet 5: 522-531, 2004.

10. Bueno MJ, Pérez de Castro I and Malumbres M: Control of cell proliferation pathways by microRNAs. Cell Cycle 7: 3143-3148, 2008.

11. Qin J and Luo M: MicroRNA-221 promotes colorectal cancer cell invasion and metastasis by targeting RECK. FEBS Lett 588: 99-104, 2014.
12. Zhang Y, Zheng L, Huang J, Gao F, Lin X, He L, Li D, Li Z, Ding Y and Chen L: MiR-124 radiosensitizes human colorectal cancer cells by targeting PRRX1. PloS One 9: e93917, 2014.

13. Zhang N, Li X, Wu CW, Dong Y, Cai M, Mok MT, Wang H, Chen J, Ng SS, Chen M, et al: microRNA-7 is a novel inhibitor of YY1 contributing to colorectal tumorigenesis. Oncogene 32: 5078-5088, 2013.

14. He X, Dong Y, Wu CW, Zhao Z, Ng SS, Chan FK, Sung JJ and $\mathrm{Yu}$ J: MicroRNA-218 inhibits cell cycle progression and promotes apoptosis in colon cancer by downregulating BMI1 polycomb ring finger oncogene. Mol Med 18: 1491-1498, 2013.

15. Wang H, An H, Wang B, Liao Q, Li W, Jin X, Cui S, Zhang Y, Ding $Y$ and Zhao L: miR-133a represses tumour growth and metastasis in colorectal cancer by targeting LIM and SH3 protein 1 and inhibiting the MAPK pathway. Eur J Cancer 49: 3924-3935, 2013.

16. Zheng YB, Luo HP, Shi Q, Hao ZN, Ding Y, Wang QS, Li SB, Xiao GC and Tong SL: miR-132 inhibits colorectal cancer invasion and metastasis via directly targeting ZEB2. World J Gastroenterol 20: 6515-6522, 2014.

17. Chen HY, Lin YM, Chung HC, Lang YD, Lin CJ, Huang J, Wang WC, Lin FM, Chen Z, Huang HD, et al: miR-103/107 promote metastasis of colorectal cancer by targeting the metastasis suppressors DAPK and KLF4. Cancer Res 72: 3631-3641, 2012.

18. Tao J, Wu D, Li P, Xu B, Lu Q and Zhang W: microRNA-18a, a member of the oncogenic miR-17-92 cluster, targets Dicer and suppresses cell proliferation in bladder cancer T24 cells. Mol Med Rep 5: 167-172, 2012.

19. Morimura R, Komatsu S, Ichikawa D, Takeshita H, Tsujiura M, Nagata H, Konishi H, Shiozaki A, Ikoma H, Okamoto K, et al: Novel diagnostic value of circulating miR-18a in plasma of patients with pancreatic cancer. Br J Cancer 105: 1733-1740, 2011.

20. Jemal A, Siegel R, Xu J and Ward E: Cancer statistics, 2010. CA Cancer J Clin 60: 277-300, 2010.

21. Lu J, Getz G, Miska EA, Alvarez-Saavedra E, Lamb J, Peck D, Sweet-Cordero A, Ebert BL, Mak RH, Ferrando AA, et al: MicroRNA expression profiles classify human cancers. Nature 435: 834-838, 2005.

22. Volinia S, Calin GA, Liu CG, Ambs S, Cimmino A, Petrocca F, Visone R, Iorio M, Roldo C, Ferracin M, et al: A microRNA expression signature of human solid tumors defines cancer gene targets. Proc Natl Acad Sci USA 103: 2257-2261, 2006.

23. Aigner A: MicroRNAs (miRNAs) in cancer invasion and metastasis: Therapeutic approaches based on metastasis-related miRNAs. J Mol Med (Berl) 89: 445-457, 2011.

24. White NM, Fatoohi E, Metias M, Jung K, Stephan C and Yousef GM: Metastamirs: A stepping stone towards improved cancer management. Nat Rev Clin Oncol 8: 75-84, 2010.

25. Xu K, Chen Z, Qin C and Song X: miR-7 inhibits colorectal cancer cell proliferation and induces apoptosis by targeting XRCC2. OncoTargets Ther 7: 325-332, 2014.

26. Tang W, Zhu Y, Gao J, Fu J, Liu C, Liu Y, Song C, Zhu S, Leng Y, Wang G, et al: MicroRNA-29a promotes colorectal cancer metastasis by regulating matrix metalloproteinase 2 and E-cadherin via KLF4. Br J Cancer 110: 450-458, 2013.

27. Zhang Y, Wang Z, Chen M, Peng L, Wang X, Ma Q, Ma F and Jiang B: MicroRNA-143 targets MACC1 to inhibit cell invasion and migration in colorectal cancer. Mol Cancer 11: 23, 2012.

28. Tsang WP and Kwok TT: The miR-18a* microRNA functions as a potential tumor suppressor by targeting on K-Ras. Carcinogenesis 30: 953-959, 2009.

29. Song BJ, Matsunaga T, Hardwick JP, Park SS, Veech RL, Yang CS, Gelboin HV and Gonzalez FJ: Stabilization of cytochrome P450j messenger ribonucleic acid in the diabetic rat. Mol Endocrinol 1: 542-547, 1987. 